\title{
Dynamics of IUD use in Vietnam: implications for family planning services at primary health care level
}

This article was published in the following Dove Press journal:

International Journal of Women's Health

I4 December 201।

Number of times this article has been viewed

\author{
Min Hae Park ${ }^{1,2}$ \\ Thang Huu Nguyen ${ }^{3}$ \\ Thoai Dinh Ngol,2 \\ 'Research and Metrics Team, Health \\ System Department, Marie Stopes \\ International; ${ }^{2}$ Faculty of Epidemiology \\ and Population Health, London School \\ of Hygiene and Tropical Medicine, \\ London, UK; ${ }^{3}$ Research and Metrics \\ Team, Marie Stopes International \\ Vietnam, Ha Noi, Vietnam
}

\begin{abstract}
This study describes the dynamics of intrauterine device (IUD) use in Vietnam and implications for family planning services. A retrospective study was conducted among women who received IUD services in 2006-2009 at six commune health stations in three provinces. Women were interviewed about IUD use and switching behaviors. Of 1316 participants, $12.1 \%$ had discontinued IUD use at 12 months after insertion, $19.4 \%$ at 24 months, and $26.9 \%$ at 36 months. The highest rates of discontinuation were among older women and farmers/manual workers. Among 434 women who had an IUD removed, 49\% cited health concerns as the main reason. Following removal, $70 \%$ switched to another contraceptive method $(n=306)$; of these, $15 \%$ switched to withdrawal, and $12 \%$ waited $>2$ months before adopting a new method. Dissatisfaction with IUD services was associated with high rates of discontinuation. Early discontinuation, delays in adopting new methods, and switching to withdrawal may contribute to unintended pregnancy among commune health station users in Vietnam.
\end{abstract}

Keywords: intrauterine device, contraceptive discontinuation, dissatisfaction

\section{Introduction}

The intrauterine device (IUD) is a long-term reversible contraceptive method that is suitable for women of all reproductive ages. ${ }^{1}$ Once inserted, the copper IUD is effective for 12 years and represents the most cost-effective reversible method. ${ }^{2}$ The IUD was used by an estimated 23\% (162 million women) of all contraceptive users in 2007, ${ }^{3}$ making it the most commonly used method after sterilization.

In Vietnam, the IUD is the only long-term reversible contraceptive method that is widely available. It is used by $52.1 \%$ of married women, accounting for more than half of all contraceptive use in the country. ${ }^{4}$ In 2002, the 12-month IUD failure rate was $3 \%$, and the 12 -month discontinuation rate was $12.5 \% .{ }^{5}$ Discontinuation of reversible methods of contraception can make a substantial contribution to unwanted pregnancies. A study that analyzed data from Demographic and Health Surveys (DHSs) from 15 developing countries estimated that discontinuation accounted for $28 \%-52 \%$ of unwanted fertility. ${ }^{6}$

Despite a contraceptive prevalence of $78 \%$ among married women, ${ }^{4}$ Vietnam has one of the highest abortion rates in the world, at 26 abortions per 1000 women (not including procedures in the private sector). ${ }^{7}$ The provision of abortion has been legal in Vietnam since the 1960s; however, the quality of reproductive health services varies greatly, and an estimated $11 \%$ of direct causes of maternal mortality are due to unsafe abortions. ${ }^{8}$ Given the prominence of the IUD in the methods mix in Vietnam, high rates of discontinuation may contribute to the country's high abortion rate. ${ }^{5}$
Correspondence: Thoai D Ngo

Research and Metrics Team-Health System Department, Marie Stopes International, I Conway Street, London, England WIT 6LP, UK

Tel +442070342352

Email thoai.ngo@mariestopes.org 
In Vietnam, free family planning (FP) services are provided through the local commune health stations (CHSs), the primary unit of the public health care system. Condoms, oral contraceptives, and IUDs are all easily obtained through the CHS. ${ }^{9}$ Perceptions of service quality play an important role in CHS use and may influence contraceptive use and continuation. Analyses of Vietnam's 1997 DHS data have found higher levels of contraceptive use in communities with increased presence of FP workers, provision of FP counseling at CHSs, and FP messages through mass media. ${ }^{10}$ Using the same data source, another study has demonstrated that risk of contraceptive discontinuation is higher in communities with low quality CHS FP services. ${ }^{11}$ There has been a paucity of research studies in recent years to understand factors influencing IUD discontinuation in Vietnam.

To address this, the present study was conducted to assess the dynamics of IUD use at the primary health care level in three Vietnamese provinces. Specifically, this study examined: (1) the sociodemographic profile of IUD users; (2) discontinuation rates and primary reasons for IUD discontinuation at 12,24 , and 36 months post-insertion; (3) switching behaviors; and (4) satisfaction level with IUD services at the CHS.

\section{Methods}

A retrospective study was conducted among women of reproductive age who had received IUD services at CHSs in Thai Nguyen, Khanh Hoa, and Vinh Long provinces between 2006 and 2009. The institutional review boards at the three provincial health departments approved the study protocol, and all study participants provided informed consent.

A multistage sampling strategy was used to select study participants. Thai Nguyen, Khanh Hoa, and Vinh Long provinces were purposively selected to represent north, central, and south regions, respectively. Thai Nguyen Province had 226,802 women of reproductive age (WRA) and 199 CHSs, Khanh Hoa had 196,383 WRA and 134 CHSs, and Vinh Long had 200,198 WRA and 101 CHSs. Within each province, two CHSs were randomly selected from a list of all facilities. To be eligible for selection, the CHSs had to have participated in government-supported FP campaigns in 2006-2009. From the selected CHSs, a list of all women who received IUD services in 2006-2009 was obtained. Women were invited to participate in the study who met the following criteria: (1) had an IUD insertion in 2006-2009; (2) aged 15-49 years at time of IUD insertion; (3) willing to give informed consent; and (4) resident in the province.
Ninety-eight percent of women on the CHS lists could be contacted. Due to strong communication networks in rural Vietnam, $100 \%$ of women who were contacted agreed to participate in the study.

An interviewer-administered questionnaire was carried out in respondents' homes. Study participants were asked about sociodemographic characteristics, IUD use, IUD discontinuation, and switching behaviors. The questionnaire was adapted from a previous study in the Philippines ${ }^{12}$ to be culturally appropriate in the Vietnamese context. Data collection took place between April and June, 2010. Data were double-entered in Epi Info Version 3.5.1 (Centers for Disease Control and Prevention, Atlanta, GA).

\section{Data analysis}

The sociodemographic characteristics of IUD users were described, and differences in discontinuation rates according to these characteristics were assessed using Pearson's chisquare test. Accounting for the variable follow-up period for each woman, Cox regression analyses were used to analyze rates of IUD discontinuation at 12, 24, and 36 months. Data were treated as single-record, single-failure data, and survival analyses were restricted to women's most recent IUD insertion. Multiple regression models were applied to estimate the effect of sociodemographic characteristics on IUD discontinuation. Statistical analyses were performed using Stata 11.1 (StataCorp LP, College Station, TX).

\section{Results Profile of IUD users}

A total of 1316 women aged 15-49 years at the time of IUD insertion were interviewed. Of these, 499 were from Thai Nguyen, 376 from Khanh Hoa, and 441 from Vinh Long provinces. The median age was 33 years (range 19-53 years; mean $33.8 \pm 7.0$ years $) ; 68 \%(n=908)$ of respondents were aged $26-40$ years, $13 \%(n=164)$ aged $\leq 25$ years, and $19 \%$ $(\mathrm{n}=244)$ aged $>40$ years (Table 1$)$.

Only $2 \%$ of the study participants $(n=26)$ had no education, while $43 \%$ had primary education $(n=568), 41 \%$ had secondary education $(n=534)$, and $14 \%$ had either high school or university education $(n=184)$. The majority of women were farmers (38\%) or housewives $(29 \%)$ ). At the time of the survey, 17 women (1\%) were not married. Half of the study participants had two children, $8 \%$ had no children, $23 \%$ had one child, and 19\% had three or more children. Data on IUD insertion were available for 1314 of the 1316 women. 
Table I Sociodemographic characteristics of 1316 women who had intrauterine devices inserted between 2006 and 2009

\begin{tabular}{ll}
\hline Characteristic & $\mathbf{N}(\%)$ \\
\hline Province & \\
Thai Nguyen & $499(37.9)$ \\
Khanh Hoa & $376(28.6)$ \\
Vinh Long & $44 I(33.5)$ \\
Age group & \\
$\leq 25$ years & $164(12.5)$ \\
$26-30$ years & $310(23.5)$ \\
3 I-35 years & $335(25.5)$ \\
$36-40$ years & $263(29.0)$ \\
$>40$ years & $244(18.5)$ \\
Education & \\
No education & $26(2.0)$ \\
Primary education & $568(43.3)$ \\
Secondary education & $534(40.7)$ \\
High school or university education & $184(14.0)$ \\
Occupation & \\
Farmer & $494(37.7)$ \\
Housewife & $377(28.8)$ \\
Worker/freelance/handcraft & $183(14.0)$ \\
Government/business/other & $257(19.6)$ \\
Marital status & \\
Married & $1,298(98.7)$ \\
Not married (divorced/separated/single) & $17(1.3)$ \\
Number of children & \\
No children & $101(7.7)$ \\
I & $305(23.2)$ \\
3-9 & $656(49.9)$ \\
Number of sons & $254(19.3)$ \\
No sons & $244(19.2)$ \\
2-7 & $707(55.7)$ \\
\hline & $276(25.1)$ \\
\hline
\end{tabular}

\section{Reasons for choosing the IUD}

Among the 1314 women with data on IUD insertion, 25\% had their most recent IUD insertion in 2006, 22\% in 2007, 22\% in 2008 , and $32 \%$ in 2009. Most women (92\%; $n=928 / 1005)$ cited convenience as the main reason for choosing an IUD, $5 \%(n=53)$ cited government encouragement, and $2 \%(n=24)$ reported other reasons (familiarity with the IUD; no desire to use oral pills). The majority of women $(90 \% ; n=901 / 1006)$ made decisions regarding choice of FP method jointly with their husbands, $9 \%$ made the decisions alone, and $1 \%$ reported that their husbands decided for them.

\section{IUD discontinuation}

Discontinuation of IUD use was $12.1 \%$ at 12 months $(95 \%$ CI 10.5-14.0), $19.4 \%$ at 24 months (95\% CI 17.4-21.7), and $26.9 \%$ at 36 months (95\% CI 24.4-29.6) (Figure 1).

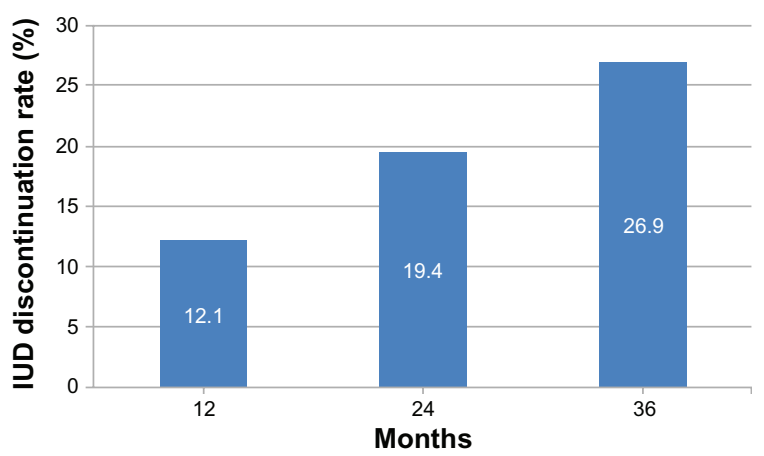

Figure I IUD discontinuation rates at I2, 24, and 36 months in all three provinces. Abbreviation: IUD, intrauterine device.

Ninety-six percent of women obtained IUD removal services at the CHS where they had the IUD inserted.

Adjusted hazard ratios (HRs) showed women in the oldest age group ( $>40$ years) had more than two times the risk of discontinuing IUD use at 12, 24, and 36 months than women aged $\leq 25$ years $(\mathrm{HR}$ for 12 -month discontinuation $=2.18$, $95 \%$ confidence interval $[\mathrm{CI}] 1.32-3.61 ; P=0.002)$. There was evidence that women who worked as housewives were less likely to discontinue IUD use than women who worked as farmers (HR at 12 months $=0.52,95 \%$ CI $0.33-0.82$; $P=0.005)$, as were women working in government or business (HR at 12 months $=0.47,95 \%$ CI $0.28-0.76 ; P=0.002$ ). There was no evidence that IUD discontinuation at 12,24 , or 36 months was associated with province, education, marital status, number of children, or number of sons (Table 2).

\section{Reasons for discontinuation}

Among the 434 women who had ever had an IUD removed, $48 \%(n=208)$ cited health concerns as the main reason for removal. Of these women, $43 \%$ reported excessive menstrual bleeding, 18\% reported weight loss, 14\% infections, and $3 \%$ discomfort during sexual intercourse. Other reasons for IUD removal included desire to switch to another FP method (14\%), expulsion (10\%), and replacement of an out-of-date IUD (17\%). Only $6 \%$ of women had their IUDs removed due to a desire for more children.

\section{Switching behaviors}

Two-thirds of women who had an IUD removed reported switching to another method $(\mathrm{n}=306 / 434)$. Of these 306 women, $28 \%$ switched to oral contraception, $17 \%$ to male condoms, $2 \%$ to injectables, $1 \%$ to sterilization, and $15 \%$ to withdrawal method. Half of all women who had an IUD removed switched to a new method within 1 week 
Table 2 Unadjusted and adjusted hazard ratios of intrauterine device discontinuation at 36 months, by sociodemographic and reproductive characteristics

\begin{tabular}{|c|c|c|c|c|}
\hline \multirow[t]{2}{*}{ Characteristics } & \multicolumn{4}{|c|}{ Discontinuation at 36 months } \\
\hline & $\begin{array}{l}\text { Unadjusted HR } \\
(95 \% \mathrm{Cl})\end{array}$ & $P$-value & $\begin{array}{l}\text { Adjusted } \mathbf{H R}^{\mathrm{a}} \\
(95 \% \mathrm{Cl})\end{array}$ & $P$-value \\
\hline \multicolumn{5}{|l|}{ Province } \\
\hline Thai Nguyen & 1.0 & & 1.0 & \\
\hline Khanh Hoa & $0.81(0.64-1.04)$ & 0.104 & $1.06(0.73-1.53)$ & 0.753 \\
\hline Vinh Long & $0.67(0.52-0.86)^{b}$ & 0.002 & $0.81(0.55-1.21)$ & 0.308 \\
\hline \multicolumn{5}{|l|}{ Age group } \\
\hline$\leq 25$ years & 1.0 & & 1.0 & \\
\hline $26-30$ years & $0.94(0.64-1.37)$ & 0.734 & $1.00(0.67-1.49)$ & 0.992 \\
\hline $31-35$ years & $0.87(0.60-1.27)$ & 0.482 & $1.06(0.7 \mid-1.59)$ & 0.764 \\
\hline $36-40$ years & $1.15(0.79-1.68)$ & 0.477 & $1.48(0.97-2.27)$ & 0.067 \\
\hline$>40$ years & $1.40(0.97-2.02)$ & 0.075 & $1.99(1.29-3.07)^{\mathrm{b}}$ & 0.002 \\
\hline \multicolumn{5}{|l|}{ Education level } \\
\hline No education/primary & 1.0 & & 1.0 & \\
\hline Secondary & $1.07(0.86-1.34)$ & 0.534 & $1.07(0.84-1.34)$ & 0.607 \\
\hline High school or university & $0.91(0.65-1.25)$ & 0.549 & $0.89(0.62-1.29)$ & 0.542 \\
\hline \multicolumn{5}{|l|}{ Occupation } \\
\hline Farmer & 1.0 & & 1.0 & \\
\hline Housewife & $0.62(0.48-0.8 \mathrm{I})^{\mathrm{b}}$ & $<0.001$ & $0.60(0.4 \mathrm{I}-0.88)^{\mathrm{c}}$ & 0.010 \\
\hline Worker/freelance/handcraft & $0.99(0.74-1.33)$ & 0.966 & $0.89(0.6|-| .3 \mid)$ & 0.564 \\
\hline Government/business/other & $0.63(0.46-0.86)^{\mathrm{b}}$ & 0.003 & $0.56(0.37-0.86)^{\mathrm{b}}$ & 0.007 \\
\hline \multicolumn{5}{|l|}{ Marital status } \\
\hline Married & 1.0 & & 1.0 & \\
\hline Not married & $0.78(0.35-1.76)$ & 0.553 & $0.82(0.36-1.87)$ & 0.642 \\
\hline \multicolumn{5}{|l|}{ Number of children } \\
\hline No children & 1.0 & & 1.0 & \\
\hline I & $0.76(0.5 I-1.13)$ & 0.173 & $1.03(0.59-1.79)$ & 0.917 \\
\hline 2 & $0.76(0.53-1.09)$ & 0.140 & $0.87(0.52-1.43)$ & 0.572 \\
\hline $3-9$ & $0.85(0.58-1.27)$ & 0.438 & $0.72(0.42-1.24)$ & 0.242 \\
\hline \multicolumn{5}{|l|}{ Number of sons } \\
\hline No sons & 1.0 & & 1.0 & \\
\hline I & $0.98(0.74-1.30)$ & 0.885 & $0.91(0.67-1.23)$ & 0.547 \\
\hline $2-3$ & $\mathrm{I} .09(0.78-\mathrm{I} .5 \mathrm{I})$ & 0.618 & $0.99(0.68-1.44)$ & 0.963 \\
\hline $4-7$ & $1.55(0.91-2.64)$ & 0.109 & $1.28(0.69-2.35)$ & 0.434 \\
\hline
\end{tabular}

Notes: ${ }^{a}$ Adjusted for all other sociodemographic and reproductive characteristics in table; ${ }^{\mathrm{b} P}<0.0 \mathrm{I}$; ${ }^{\mathrm{c} P}<0.05$.

Abbreviations: $\mathrm{HR}$, hazard ratio; $\mathrm{Cl}$, confidence interval.

of removal ( $\mathrm{n}=190 / 382), 10 \%$ switched after 2 weeks, $28 \%$ after 1 month, and $12 \%$ waited $\geq 3$ months. A smaller proportion of young women switched to a different method after IUD discontinuation compared with older women ( $53 \%$ aged $\leq 25$ years versus $66 \%$ aged $>40$ years; $P=0.005$ ). Older women were less likely to switch to a modern method (83.1\% aged $>40$ years versus $95.6 \%$ aged $\leq 25$ years). Health effects were the most commonly cited reasons for choosing a different contraceptive method.

\section{Satisfaction with the IUD and IUD services}

Ninety-eight percent of women $(n=1283 / 1316)$ stated that they would recommend the IUD to friends or relatives. When asked about IUD services, 91\% of women $(n=1192)$ were satisfied or very satisfied with IUD services at the CHS, while $8 \%(n=105)$ felt neutral and only $1 \%(n=11)$ were not satisfied. Ninety-six percent of respondents $(n=1252)$ reported that they would use IUD services at their CHS if they needed a FP method in the future.

IUD discontinuation at 12, 24, and 36 months was strongly associated with measures of satisfaction. Among women who were not satisfied with the IUD services at the CHS, 91\% had discontinued the method at 36 months, compared with $23 \%$ of women who were satisfied or very satisfied $(P=0.001)$. Among women who would not readily use IUD services at the CHS if they needed contraceptives, 73\% had discontinued use at 36 months, compared with 19\% of those who said they would readily use the services again $(P<0.001)$. Seventy-nine percent of women who would not recommend the IUD to others had discontinued use by 
36 months, compared with $21 \%$ of those who would readily recommend the method $(P<0.001)$. Women who were satisfied with the IUD services were more likely to switch to a modern method of contraception than those women who were not satisfied ( $87 \%$ versus $73 \% ; P=0.003$ ).

\section{Discussion}

This study has shown that in Thai Nguyen, Khanh Hoa, and Vinh Long provinces in Vietnam, 27\% of women discontinued IUD use within 3 years after insertion. The discontinuation rates reported in this study are consistent with the rates reported in the 2002 Demographic Health Survey (DHS), which estimated IUD discontinuation in Vietnam at $12.5 \%$ at 12 months, $20.4 \%$ at 24 months, and $30.6 \%$ at 36 months. ${ }^{13}$ This is comparable to respective rates of $9.6 \%$, $17.7 \%$, and $30.8 \%$ in Turkey, and $11.4 \%, 22.3 \%$, and $30.5 \%$ in Kazakhstan, countries in which the IUD represents an important long-term family planning method (prevalence of IUD use $20 \%$ in Turkey and $42 \%$ in Kazakhstan). ${ }^{13}$

Early discontinuation among older women may be attributable to reaching menopause, but the finding that women in farming/manual occupations were more likely to discontinue IUD use has implications for FP campaigns. Women who work as farmers or in manual occupations may represent potential target groups for FP campaigns to improve IUD continuation. Although we do not have data on the rural/ urban status of women, it is likely that women who work as farmers live in rural areas, and it is known that women living in rural areas are more likely to access public health services than women living in urban areas. ${ }^{14}$ DHS data show that $87 \%$ of IUD episodes are in rural women, compared with $75 \%$ of other modern method episodes; ${ }^{13}$ this may reflect more limited contraceptive options in rural areas, ${ }^{9}$ an issue which should be addressed by providers.

Despite the fact that just $6 \%$ of study respondents removed their IUD due to the desire to have children, one-third of women did not switch to another method after IUD removal. Fifty percent of women waited for more than a week before switching to an alternative method, and $12 \%$ waited longer than 2 months. This delay in adopting new methods of contraception poses the risk for unintended pregnancy following IUD discontinuation. In the United States, it has been shown that more than half of unintended pregnancies occur within a month when contraceptives are not used. ${ }^{15}$ Furthermore, in the present study, $15 \%$ of women who switched methods began using withdrawal, which is an unreliable method (United States data indicate a 12-month failure rate of $18 \%{ }^{16}$ ). Delays to switching may point to a gap in FP services, and a need for improved contraceptive counseling and access to alternative contraceptive methods. While the IUD has dominated the contraceptive methods mix in Vietnam for many years, the growing role of private providers in $\mathrm{FP}^{17}$ may present opportunities for widening contraceptive choice and access to alternative methods for women. Recent data from Vietnam have shown an increasing role of alternative methods in the contraceptive mix; between 2002 and 2010, the proportion of contraceptive use that was accounted for by the oral pill rose from $10.5 \%$ to $15.5 \%$, while for the male condom this figure increased from $8.4 \%$ to $13.1 \%{ }^{4}$

The majority of women in this study discontinued IUD use or switched to alternative methods due to health concerns. Similar findings were reported in a study of six developing countries, which showed that health concerns were the most common reason for discontinuation of the IUD and hormonal methods. ${ }^{18}$ Further research is required to explore whether more effective post-IUD insertion care could improve continuation in this setting; experiences of IUD campaigns in other countries have highlighted that high-quality follow-up care is essential for instilling women's confidence in the method. ${ }^{19}$

In this study, dissatisfaction with IUD services was strongly associated with discontinuation. Due to the small number of women who were dissatisfied with IUD services $(n=11)$, this result should be interpreted with caution. However, the finding is consistent with the findings of a study of the Vietnam 1997 DHS data, which showed that women living in communities with high-quality health centers were less likely to discontinue use of modern contraceptive methods. ${ }^{11}$ Due to the retrospective nature of this study, it is not possible to establish the direction of causality, and it is likely that women who experienced problems with their IUD were more likely to be critical of services than other women. To establish the nature of the relationship between perceived quality of services and IUD discontinuation, prospective studies are needed. Further examination of the reasons for women's dissatisfaction with IUD services could identify ways to improve service quality and IUD continuation; qualitative information on women's experiences of IUD services would provide a more comprehensive picture of the situation. Despite these limitations, the picture that emerged from this study was that the majority of women were satisfied with their experience of IUD services at the CHS.

There are other limitations to this study. Notably, estimates of IUD use duration were reported to the nearest year, and more detailed information (eg, discontinuation 
at 3 or 6 months) was unavailable. Furthermore, data were self-reported and subject to recall bias. Women reported on only their most recent IUD removal, therefore not all episodes of IUD use during 2006-2009 could be considered. Consequently, some of the more complex patterns of IUD use, eg, use of IUDs to space or stop births, were unable to be discerned. The use of contraceptive calendars would generate more complete data on patterns of childbearing and IUD use. Finally, the sampling strategy used means that the findings may not be generalizable to the wider Vietnamese population, although the findings appear to be consistent with those from nationally representative DHS data.

This was one of the first community-based studies focusing on the dynamics of IUD use in Vietnam, and provides updated data on IUD discontinuation. Early IUD discontinuation, delays to adopting new methods, and switching to unreliable methods may contribute to unintended pregnancy in Vietnam. Greater emphasis on effective FP counseling and follow-up care, and widening access to alternative contraceptive methods, could strengthen Vietnam's FP services.

\section{Acknowledgments}

This study was funded by the Research and Metrics Team at Marie Stopes International. The Departments of Health (DOH) in Thai Nguyen, Khanh Hoa, and Vinh Long provinces, Vietnam supported the implementation of this study. The authors wish to thank Professor John Cleland at the London School of Hygiene and Tropical Medicine for his critical reading and discussion of this manuscript. MHP, THN, and TDN work for Marie Stopes International, an organization that provides sexual reproductive health and family planning services globally.

\section{Disclosure}

MHP, THN, and TDN work for Marie Stopes International, an organization that provides sexual reproductive health and family planning services globally and in Vietnam. The opinions expressed in this manuscript are the views of the authors, and do not reflect the views and opinions of their employers or funding bodies.

\section{References}

1. Deans EI, Grimes DA. Intrauterine devices for adolescents: a systematic review. Contraception. 2009;79(6):418-423.

2. Chiou CF, Trussell J, Reyes E, et al. Economic analysis of contraceptives for women. Contraception. 2003;68(1):3-10.

3. United Nations. World Contraceptive Use 2009. United Nations; 2009.

4. Vietnam General Statistics Office. The 1/4/2010 population change and family planning survey: major findings. Hanoi: Vietnam General Statistics Office; 2011.

5. Committee for Population, Family and Children [Vietnam], ORC Macro. Vietnam Demographic and Health Survey 2002. Calverton, MD: Committee for Population, Family and Children and ORC Macro; 2003.

6. Blane A, Curtis S, Croft T. Does contraceptive discontinuation matter? Quality of care and fertility consequences. In: MEASURE Evaluation Technical Report No. 3. University of North Carolina at Chapel Hill: Carolina Population Center; 1999.

7. Sedgh G, Henshaw SK, Singh S, Bankole A, Drescher J. Legal abortion worldwide: incidence and recent trends. Perspect Sex Reprod Health. 2007;39(4):216-225.

8. Vietnam Ministry of Health and Maternal and Child Health and Family Planning Department. Vietnam Ministry of Health, Maternal and Child Health and Family Planning Department, Research on Maternal Mortality in Vietnam, 2000-2001. Hanoi: Vietnam Ministry of Health; 2003.

9. Thang NM, Anh DN. Accessibility and use of contraceptives in Vietnam. Int Fam Plan Perspect. 2002;28(4):214-219.

10. Thang NM, Huong VT. Changes in contraceptive use in Vietnam. J Biosoc Sci. 2003;35(4):527-543.

11. Do MP, Koenig MA. Effect of family planning services on modern contraceptive method continuation in Vietnam. J Biosoc Sci. 2007;39(2):201-220.

12. Ngo TD, Pernito VL. Discontinuation of IUDs among women receiving mobile outreach services in the Philippines, 2006-2008. London: Marie Stopes International; 2009.

13. Ali MM, Sadler RK, Cleland J, Ngo TD. Long-term contraceptive protection, discontinuation, and switching behaviour: intrauterine device (IUD) use dynamics in 14 developing countries. London: World Health Organization and Marie Stopes International; 2011.

14. Ha NTH, Berman P, Larsen U. Household utilization and expenditure on private and public health services in Vietnam. Health Policy Plan. 2002;17(1):61-70.

15. Finer LB, Henshaw SK. Disparities in rates of unintended pregnancy in the United States, 1994 and 2001. Perspect Sex Reprod Health. 2006;38(2):90-96.

16. Kost K, Singh S, Vaughan B, Trussell J, Bankole A. Estimates of contraceptive failure from the 2002 National Survey of Family Growth. Contraception. 2008;77(1):10-21.

17. Park MH, Nguyen TH, Dinh TH, Ngo TD. Government social franchising for reproductive healthcare in Vietnam. London: Marie Stopes International; 2009.

18. Ali M, Cleland J. Contraceptive discontinuation in six developing countries: a cause-specific analysis. Int Fam Plan Perspect. 1995;21: 92-97.

19. d'Arcangues C. Worldwide use of intrauterine devices for contraception. Contraception. 2007;75(6 Suppl 1):S2-S7.
International Journal of Women's Health

\section{Publish your work in this journal}

The International Journal of Women's Health is an international, peerreviewed open-access journal publishing original research, reports, reviews and commentaries on all aspects of women's healthcare including gynecology, obstetrics, and breast cancer. Subject areas include: Chronic conditions (migraine headaches, arthritis, osteoporosis);

\section{Dovepress}

Endocrine and autoimmune syndromes; Sexual and reproductive health; Psychological and psychosocial conditions. The manuscript management system is completely online and includes a very quick and fair peer-review system. Visit http://www.dovepress.com/ testimonials.php to read real quotes from published authors. 